\title{
Une nouvelle zone de neurogenèse fonctionnelle
}

$>$ En dehors de deux structures précises: la zone sous-granulaire et la zone sous-ventriculaire, le cerveau du mammifère adulte est considéré comme non neurogène, c'est-à-dire incapable de produire de nouveaux neurones. Cependant, les influences environnementales qui s'opposent à cette activité peuvent être mises entre parenthèses dans certaines conditions. C'est ce qui se passe lorsque l'on réalise une neurectomie vestibulaire unilatérale chez le chat adulte. Nos travaux révèlent en effet l'existence d'une neurogenèse réactionnelle dans les noyaux vestibulaires désafférentés situés dans le tronc cérébral. Plus étonnant encore, nous avons montré que cette prolifération cellulaire postlésionnelle était fonctionnelle et participait aux processus de restauration des fonctions posturolocomotrices. <

\section{Le renouvellement des neurones dans le cerveau adulte}

Au même titre que les cellules intestinales ou sanguines, de nouveaux neurones sont capables d'être générés tout au long de la vie au sein de leur tissu d'appartenance. Ainsi, contrairement au dogme érigé en 1928 par Ramon y Cajal $^{1}$ : «Une fois le développement terminé, les sources de la croissance et de la régénération des axones et des dendrites sont taries de manière irrévocable. Dans le cerveau adulte, les voies nerveuses sont fixées et immuables : tout peut mourir, rien ne peut régénérer... », il est désormais communément admis qu'une fois le développement achevé, le cerveau ne se fige pas en une entité rigide. À l'âge adulte, le système nerveux central (SNC) reste un organe d'une extrême malléabilité qui s'adapte sans cesse à son environnement. De nombreux progéniteurs neuraux (nous utiliserons le terme progéniteur pour qualifier indifféremment les cellules souches neurales et leurs descendants immédiats) sont disséminés dans le

${ }^{1}$ Ramon y Cajal, S (1928). Degeneration and regeneration of the nervous system vol. 2. New York, USA, Haffner Publishing Co, p. 750.

\section{Les noyaux vestibulaires du tronc cérébral}

Sophie Dutheil, Michel Lacour, Brahim Tighilet

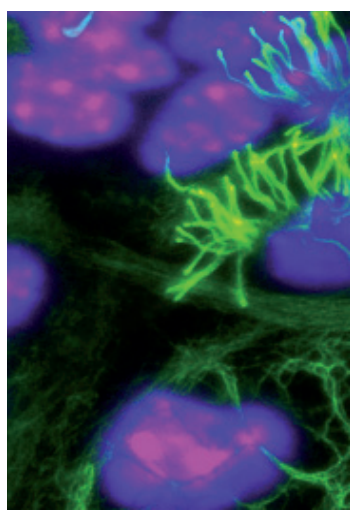

UMR 6149,

Neurosciences

intégratives et adaptatives, pôle 3C : Comportement cerveau, cognition, Centre de Saint-Charles, case B, 3, place Victor Hugo, 13331 Marseille Cedex 3, France. brahim.tighilet@univ-provence.fr

SNC du mammifère adulte. Toutefois, à ce jour, seulement deux structures bien précises, la zone sous-granulaire du gyrus dentelé de l'hippocampe et la zone sous-ventriculaire qui borde les ventricules latéraux, renouvellent continuellement leur stock de neurones (Figure 1). Ces zones sont dites de neurogenèse car elles génèrent de nouveaux neurones [39]. La grande majorité d'entre eux survit et s'intègre ensuite dans des circuits neuronaux qui contribuent à la transmission de l'information dans le cerveau : tâches d'apprentissage, de mémorisation, d'orientation spatiale, ou encore de reconnaissance olfactive [1].

Par contraste, tout le reste du SNC est considéré comme étant non neurogène car les progéniteurs neuraux potentiellement présents sont maintenus dans un état de quiescence. L'environnement ne permet pas la prolifération, la survie et la différenciation cellulaires. Ainsi, des progéniteurs neuraux de la zone sous-granulaire ou de la zone sousventriculaire transplantés dans des zones non neurogènes meurent ou s'y différencient uniquement en cellules gliales [2-4]. En revanche, lorsque des progéniteurs provenant de n'importe quelle région du SNC sont transplantés dans des zones de neurogenèse, ils s'y différencient selon le type neuronal requis par le tissu hôte, neurones granulaires dans la zone sous-granulaire et interneurones dans la zone sous-ventriculaire [3-6]. Il apparaît donc que le devenir des progéniteurs neuraux au sein de leur tissu hôte est avant tout commandité par l'environnement extracellulaire, au détriment des paramètres intrinsèques aux cellules $[7,8]$. De manière intéressante, les influences qui semblent s'opposer à la neurogenèse dans la quasi-totalité du SNC pourraient être ponctuellement mises entre parenthèses dans certaines conditions. En effet, de nombreux travaux chez le mammifère adulte ont révélé que des pathologies particulières (ischémie, scléroses multiples, 


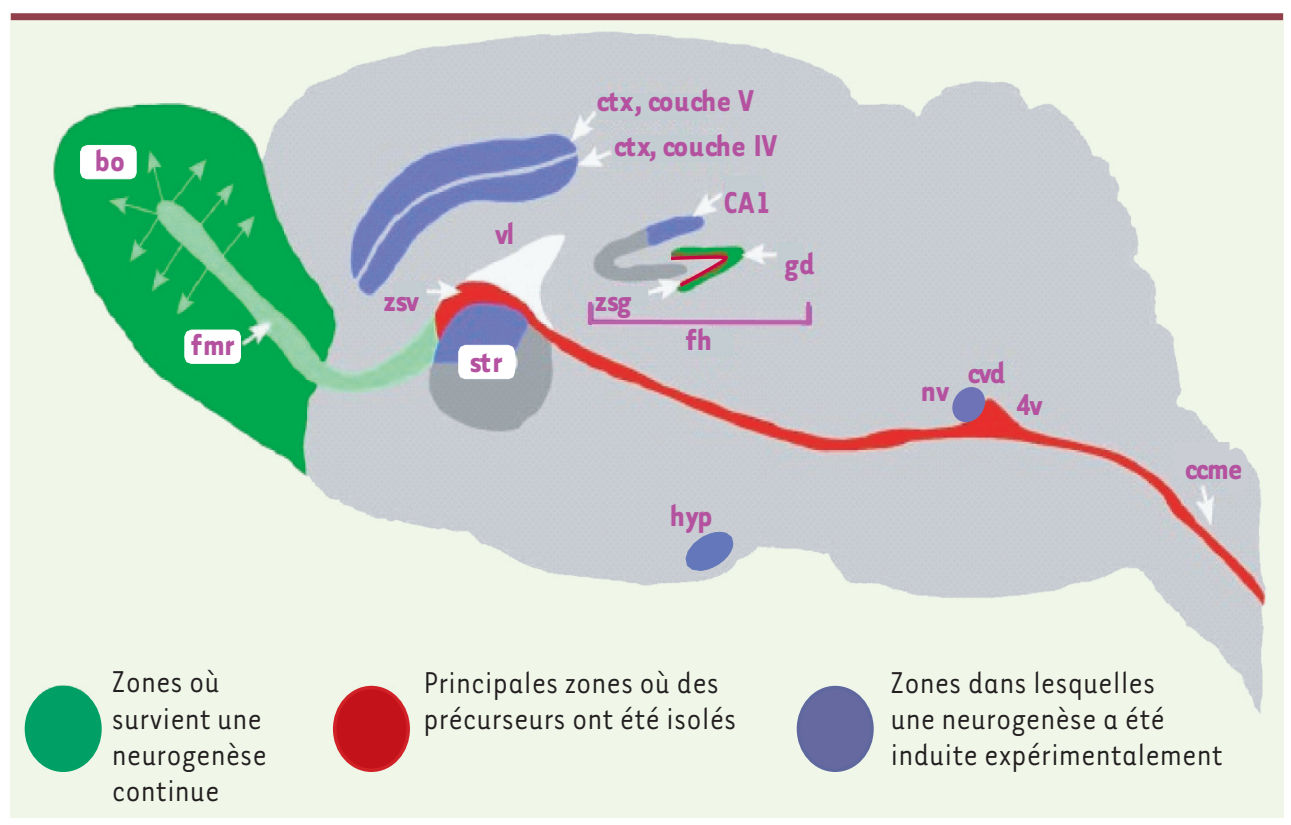

Figure 1. Les différentes structures cérébrales contenant des progéniteurs neuraux, une neurogenèse continue et une neurogenèse induite expérimentalement dans le cerveau du mammifère adulte. Schéma représentant les deux régions où survient une neurogenèse continue chez le mammifère adulte (vert : zone sous-ventriculaire/bulbe olfactif et gyrus dentelé de I'hippocampe) et les principales régions où des populations de progéniteurs neuraux ont été identifiées (rouge: zone sous-granulaire du gyrus dentelé et neuraxe rostro-caudal qui s'étend de la

zone sous-ventriculaire antérieure jusqu'au canal central de la moelle épinière). Une neurogenèse limitée induite expérimentalement a également été mise en évidence dans d'autres régions du système nerveux central (violet : noyaux vestibulaires, complexe vagal dorsal, couches IV et $V$ du cortex, aire CAl, striatum, hypothalamus). Certaines régions dans lesquelles l'existence d'une neurogenèse est controversée ont été omises par souci de simplicité. ccme : canal central de la moelle épinière ; ctx : cortex ; cvd : complexe vagal dorsal ; fh : formation hippocampique ; gd : gyrus dentelé de l'hippocampe ; hyp : hypothalamus ; vl : ventricule latéral ; bo : bulbe olfactif ; fmr : flux migratoire rostral ; zsg : zone sous-granulaire (du gyrus dentelé) ; zsv : zone sous-ventriculaire ; str : striatum ; $4 \mathrm{v}$ : IVe ventricule ; nv : noyaux vestibulaires (modifié d'après [7]).

chorée de Huntington, maladie d'Alzheimer) ou certaines lésions s'accompagnaient d'une neurogenèse réactionnelle dans des zones du SNC considérées jusque-là comme incapables de produire de nouveaux neurones [8, 9] (Figure 1). Sous l'influence de signaux spécifiques, un tissu considéré comme non neurogène peut donc s'affranchir des restrictions endogènes et permettre une prolifération cellulaire limitée dans le temps pour renouveler son stock neuronal local.

\section{Naissance et intégration de nouveaux neurones dans les noyaux vestibulaires après lésion vestibulaire}

\section{Atteinte vestibulaire et compensation vestibulaire}

Les fonctions vestibulaires sont assurées par des informations captées de manière symétrique par des récepteurs vestibulaires situés dans l'oreille interne : les canaux semicirculaires et les otolithes $^{2}$. Ces deux types de récepteurs sont sensibles respectivement aux accélérations angulaires et linéaires (incluant le vecteur gravitaire). Les messages véhiculés par les nerfs vestibulaires convergent d'abord vers les noyaux vestibulaires, transitent par des relais thalamiques pour achever leur trajet dans un ensemble d'aires corticales (cortex vestibulaire pariéto-insulaire, aires $3 a$ et $2 v$ ). Le traitement et l'intégration de ces signaux vestibulaires et d'autres signaux issus de modalités sensorielles différentes (proprioception

${ }^{2}$ L'organe récepteur comprend des cellules ciliées dont les cils sont emprisonnés dans une substance gélatineuse contenant des petits cristaux appelés otolithes. et vision en particulier) gèrent le contrôle de la posture et de la locomotion, de la stabilisation du regard et de l'orientation spatiale. Une atteinte vestibulaire unilatérale résultant de lésions traumatiques, d'atteintes virales (névrite) ou encore d’hydrops (maladie de Ménière) engendre systématiquement une quadruple symptomatologie aussi bien chez l'homme que chez de nombreuses espèces. Ce syndrome comprend des désordres posturo-locomoteurs, oculomoteurs, végétatifs et cognitifs (Figure 2) dont l'expression diminue progressivement avec le temps grâce au phénomène de «compensation vestibulaire »[10]. Lorsque les désordres vestibulaires ne se compensent toutefois pas d'eux-mêmes et que les patients atteints de la maladie de Menière ne répondent pas aux traitements pharmacologiques, la pratique d'une neurectomie vestibulaire unilatérale ${ }^{3}$ (NVU) permet de les soulager définitivement de leurs crises vertigineuses.

Nos travaux de recherche sont consacrés depuis plusieurs années à la compensation vestibulaire qui représente un excellent modèle d'étude des phénomènes de plasticité post-lésionnelle dans le système nerveux central. Les déficits statiques observés au stade aigu d'une lésion

\footnotetext{
${ }^{3}$ II s'agit de la section du nerf vestibulaire.
} 
Symptômes perceptivo-cognitifs Désorientation spatiale Vertiges

Altération de la verticale subjective

Projection des noyaux vestibulaires vers:

Noyau du tractus solitaire

Areas postrema

Noyau dorsal du vague Noyau parabrachial

\section{Symptômes} végétatifs Vomissements Nausées Salivation

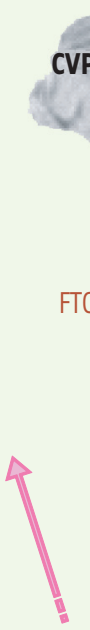

FTC $3 a \quad 2 v$

Noyaux
vestibulaires
controlatéraux
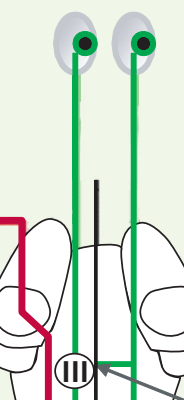

Symptômes oculomoteurs

Cyclotorsion

Nystagmus

Réflexe vestibulo-oculaire altéré

Noyaux oculomoteurs

Noyaux vestibulaires ipsilatéraux

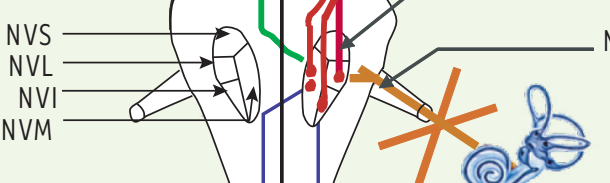

Nerf vestibulaire

Récepteurs vestibulaires de l'oreille interne

\section{Symptômes posturo-locomoteurs}

Tonus musculaire asymétrique

Réflexes vestibulo-spinaux altérés

Surface du polygone de sustentation élargie

Figure 2. Organisation anatomo-fonctionnelle du complexe des noyaux vestibulaires (NV). Le nerf vestibulaire contacte les cellules sensorielles des récepteurs labyrinthiques périphériques (canaux semi-circulaires, utricule et saccule) et se projette ipsilatéralement sur les 4 différents noyaux vestibulaires (NV) : médian (NVM), inférieur (NVI), latéral (NVL) et supérieur (NVS). Les NV sont localisés dans la partie dorso-latérale de la jonction bulbo-protubérantielle du tronc cérébral, sous le plancher du IVe ventricule. Ils forment le premier relais des réflexes vestibulo-oculaires, vestibulo-spinaux, vestibulo-végétatifs et des voies ascendantes vestibulo-corticales impliquées dans l'orientation spatiale. Les NV sont à l'origine de messages prémoteurs (commande de la musculature oculaire et somatique) au service de la régulation de la posture et de la stabilisation du regard. Ils projettent, par le faisceau longitudinal médian (FLM) sur les noyaux oculomoteurs (noyau oculomoteur III, noyau trochléaire ou abducens IV) dont les motoneurones commandent la musculature oculaire afin de produire des réactions compensatoires de l'œil stabilisant les images sur la rétine lors de mouvements de la tête. Les projections vestibulo-spinales, originaires du NVL ipsilatéral, atteignent tous les étages médullaires via le faisceau vestibulo-spinal latéral (FVSL). Les projections controlatérales des NV médian, inférieur et latéral constituent le faisceau vestibulo-spinal médian (FVSM) et commandent les motoneurones du cou et de la partie supérieure de l'axe du corps. Les NV reçoivent des afférences cérébelleuses, médullaires (proprioceptives), visuelles (optocinétiques), des noyaux vestibulaires controlatéraux et des aires corticales. Chez les mammifères supérieurs, il existe plusieurs aires corticales plurisensorielles impliquées dans la réception des messages vestibulaires (aire $3 a$, aire 2v, cortex vestibulaire pariéto-insulaire) cheminant par le FTC (faisceau thalamo-cortical).

vestibulaire (nystagmus oculaire ${ }^{4}$, inclinaison de la tête du côté lésé, asymétrie du tonus des muscles des membres, élargissement de la surface de sustentation, nausées) (Figure 2) résultent de l'asymétrie de l'activité électrique spontanée entre les noyaux vestibulaires (NV)

${ }^{4}$ Le nystagmus est un mouvement involontaire et saccadé du globe oculaire qui comprend deux composantes, une dérive lente et une secousse de rappel rapide. II est défini par le sens de sa secousse rapide. homologues situés dans le tronc cérébral. En effet, consécutivement à l'atteinte vestibulaire, les NV ipsilatéraux à la lésion deviennent silencieux tandis que les NV controlatéraux conservent une activité électrique. Ce déséquilibre électrophysiologique qui se réajuste peu à peu avec une constante de temps variable selon les espèces est considéré comme le substrat neurophysiologique 


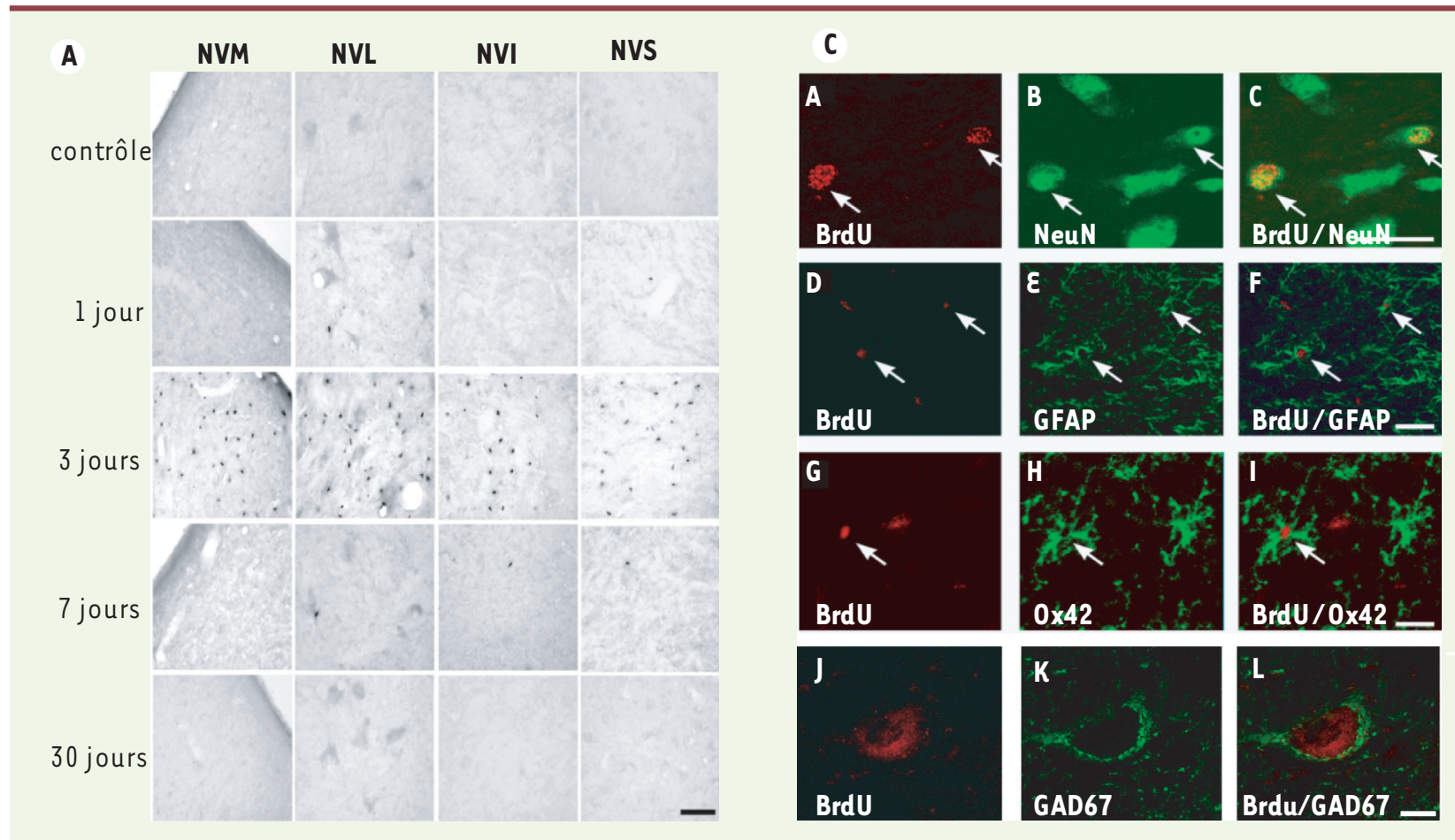

B
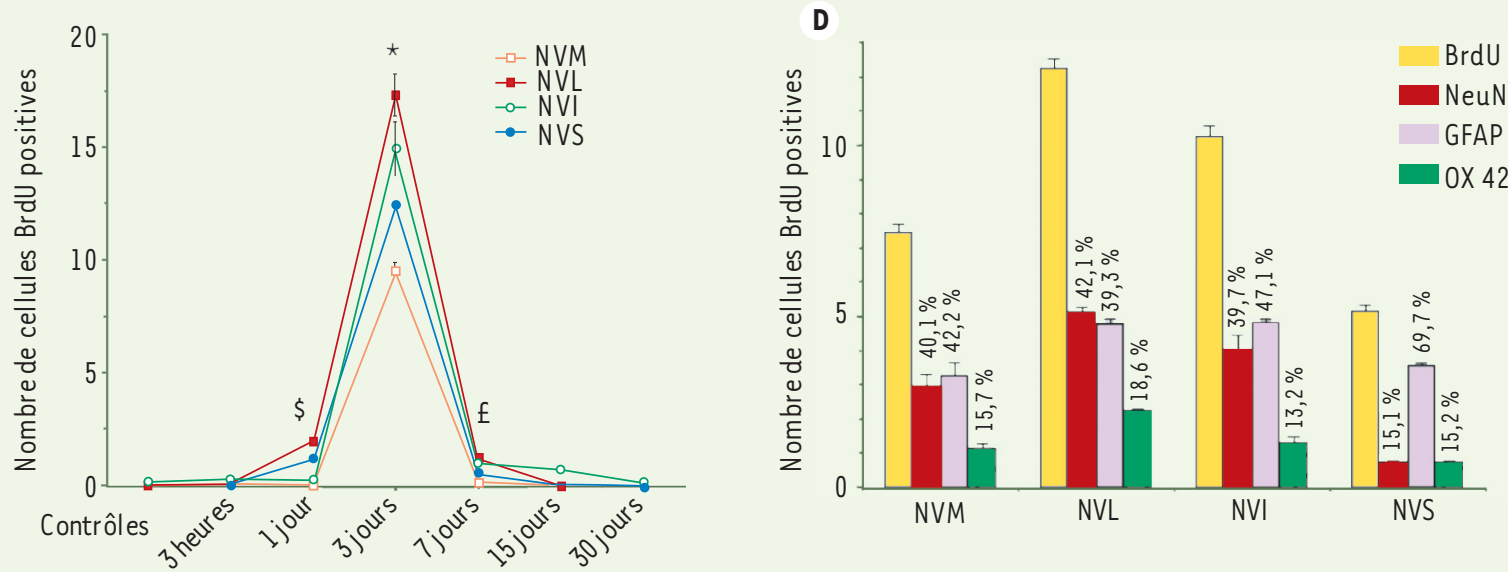

Cinétique de la prolifération cellulaire après NVU

de la compensation vestibulaire $[11,12]$. À l'inverse, les déficits dynamiques comme l'altération du gain du réflexe vestibulo-oculaire ${ }^{5}$ lors d'un mouvement de la tête du côté lésé ne sont pas compensés, ou seulement partiellement et avec une constante de temps plus longue [13]. Une partie des processus responsables de ce retour à l'équilibre électrophysiologique entre les NV ipsi- et controlatéraux a été attribuée à des mécanismes de neuroplasticité survenant dans les NV : réorganisations moléculaires et neurochimiques pré- et post-synaptiques, pousses neuritiques, réactions astrocytaires et microgliales, chan-

${ }^{5}$ Le réflexe vestibulo-oculaire stabilise le regard, c'est-à-dire compense les mouvements de la tête pou permettre à l'homme ou à l'animal de voir clairement l'environnement dans lequel sa tête tourne. Pendant une rotation de la tête, le RVO imprime à l'œil une rotation d'amplitude et de vitesse égales mais de sen opposé à celle de la tête, de sorte que l'axe visuel est maintenu stable dans l'espace. gement de sensibilité des récepteurs membranaires, régulation du nombre de récepteurs membranaires, libération de neuromédiateurs et de neurohormones [14-19]. Le SNC fait donc preuve d'une extraordinaire capacité d'adaptation post-lésionnelle qui met en jeu un ensemble de mécanismes qui s'échelonnent à tous niveaux, du moléculaire au comportemental.

Induction d'une zone de neurogenèse après neurectomie vestibulaire unilatérale

Dans notre modèle expérimental de NVU chez le chat adulte (technique décrite dans l'encadré), de fortes augmentations du nombre de neurones exprimant les 
$\triangle$ Figure 3. Décours temporel de la prolifération cellulaire dans les noyaux vestibulaires désafférentés après neurectomie vestibulaire unilatérale chez le chat adulte A. Microphotographies illustrant le marquage BrdU immunopositif (BrdU ${ }^{+}$) dans les noyaux vestibulaires (NV) de chats contrôles et dans les NV désafférentés de chats examinés 1, 3, 7 et 30 jours après une neurectomie vestibulaire unilatérale (NVU). Chez les animaux contrôles aucune cellule BrdU ${ }^{+}$'est présente dans les NV. Échelle : $100 \mu \mathrm{m}$. B. Quantification du nombre de cellules néoformées marquées au BrdU dans le complexe des noyaux vestibulaires (NV) du côté de la NVU. Par rapport à des animaux contrôles chez qui aucune cellule en division mitotique n'est observée dans les NV, un pic de prolifération cellulaire apparaît 3 jours après la NVU dans les 4 principaux NV : le latéral (NVL), I'inférieur (NVI), le médian (NVM) et le supérieur (NVS). La prolifération cellulaire est quasiment absente dès la fin de la $1^{\text {re }}$ semaine post-lésionnelle. $C$. Différenciation cellulaire dans les noyaux vestibulaires désafférentés après neurectomie vestibulaire unilatérale chez le chat adulte. L'utilisation de marqueurs spécifiques des cellules microgliales (0x42, anticorps monoclonal anti-CD1lb/c), des astrocytes (GFAP, glial fibrillary acidic protein) et des neurones (NeuN) permet de déterminer la nature des cellules en prolifération (BrdU). L'analyse en microscopie confocale montre que des nouvelles cellules ont incorporé du BrdU 3 jours après NVU, ont survécu et se sont différenciées en neurones, en astrocytes et en microglie 30 jours après la lésion. Les noyaux $B r d U^{+}$sont en rouge et les autres marqueurs de différenciation en vert : $\mathrm{NeuN}^{+}(B, C) ; G F A P^{+}(\varepsilon, F) ; 0 \times 42^{+} ;(H, I) ; G A D 67^{+}(K, L)$. Un nouveau neurone GABAergique appartenant au noyau vestibulaire médian désafférenté est illustré (J, K, L). Le marquage nucléaire au BrdU ( $\mathrm{J}$ ) indique qu'il s'agit d'une nouvelle cellule qui a survécu à ce délai post-lésionnel ; le marquage GAD67 (K) montre qu'elle exprime l'enzyme de synthèse du GABA. La colocalisation par cette cellule des 2 marqueurs ( $L$ ) démontre qu'il s'agit d'un neurone GABAergique de moyenne taille (barre de calibration : $20 \mu \mathrm{m}$ ). D. Histogrammes représentant le nombre de cellules $\mathrm{BrdU}^{+}, \mathrm{NeuN}^{+}, \mathrm{GFAP}^{+}$et $0 \times 42^{+}$dans les NV 30 jours après NVU. Les pourcentages indiqués sont calculés dans la population BrdU+. L'analyse des colocalisations en microscopie confocale montre qu'un fort pourcentage de cellules se différencient en neurones (environ $40 \%$ ) ou en astrocytes (pourcentage similaire) dans les noyaux vestibulaires latéral (NVL), inférieur (NVI) et médian (NVM), tandis que le taux de neurones est très faible dans le noyau supérieur (NVS) qui, en revanche, a un fort taux d'astrocytes. On remarquera que la proportion de cellules microgliales est faible et comparable (environ $15 \%$ ) dans tous les noyaux vestibulaires.

phénotypes GABAergique [20] et cholinergique [21] ont été observées dans les NV du côté ipsilatéral à la lésion. Ces résultats ont été interprétés comme reflétant des changements de phénotypes neuronaux concomitants de la régulation positive d'enzymes nécessaires à la synthèse de GABA et d'acétylcholine. Cependant, une nouvelle hypothèse a été avancée en 2002 avec la découverte de progéniteurs neuraux au niveau du plancher du IVe ventricule. En effet, si l'on administrait localement des facteurs de croissance, ces cellules devenaient capables de générer de nouveaux neurones in vivo [22]. De plus, une prolifération cellulaire consécutive à une vagotomie a été mise en évidence dans le complexe dorsal du vague, à proximité des NV [23]. Ces résultats nous ont conduits à postuler que l'accroissement du nombre de neurones GABAergiques et cholinergiques observé après NVU pouvait résulter d'une prolifération cellulaire.

Pour confirmer cette hypothèse, nous avons utilisé un marqueur des cellules en division : le bromodéoxyuridine (BrdU), un analogue de la thymidine qui s'incorpore dans l'ADN des cellules lors de la phase $S$ du cycle cellulaire. Cet agent est généralement utilisé pour dater les nouvelles cellules et évaluer la prolifération cellulaire. Nous avons démontré l'existence d'une forte prolifération de cellules ayant incorporé le $\mathrm{BrdU}\left(\mathrm{BrdU}^{+}\right)$uniquement dans les NV situés du côté de la lésion (Figures $3 A$ et $B$ ), avec un pic culminant 3 jours après la lésion $[24,25]$. Des travaux en cours indiquent également un pic d'expression de neurotrophines (brain-derived neurotrophic factor [BDNF] et nerve growth factor $[\mathrm{NGF}]$ ), agents proneurogènes favorisant la survie et la différenciation cellulaires, après ce même délai postlésionnel. L'étude de la cinétique des nouvelles cellules formées en fonction du temps post-lésionnel a indiqué qu'une majorité survivait au-delà d'un mois - la proportion variant selon les différents NV (60 à $70 \%$ en moyenne). Certaines de ces cellules se sont différenciées en cellules gliales (astrocytes, microglie, voir Figure $3 C$ et $D$ ), confirmant la réaction gliale déjà décrite par d'autres auteurs chez le rat [14]. D'autres cellules $\mathrm{BrdU}^{+}$exprimaient aussi le marqueur NeuN (neuronal nuclei) spécifique des neurones matures, mettant ainsi en évidence la différenciation des nouvelles cellules en neurones (Figure $3 C$ ). Ces résultats ont ainsi démontré pour la première fois la naissance de nouveaux neurones dans les NV désafférentés après une lésion vestibulaire chez l'animal adulte.

Nous avons par la suite caractérisé le phénotype de ces nouveaux neurones et trouvé qu'ils exprimaient la GAD67 (glutamic acid decarboxylase 67), enzyme de synthèse du GABA. Les données recueillies en microscopie confocale ont mis en évidence une colocalisation des deux marqueurs BrdU et GAD67 (Figure 3C) pour certains de ces neurones, démontrant l'existence d'une neurogenèse de type GABAergique dans les NV du côté de la lésion. Ce résultat est à rapprocher d'observations que nous avons faites antérieurement [20] montrant chez le chat une augmentation du nombre de neurones exprimant le GABA après NVU. De manière intéressante, nous n'avons pas observé de neurones cholinergiques parmi les cellules BrdU'. L'absence d'une neurogenèse cholinergique suggère que l'augmentation du nombre de neurones cholinergiques dans les NV désafférentés résulterait d'un changement de phénotype neuronal [21]. Ce résultat original témoigne vraisemblablement 


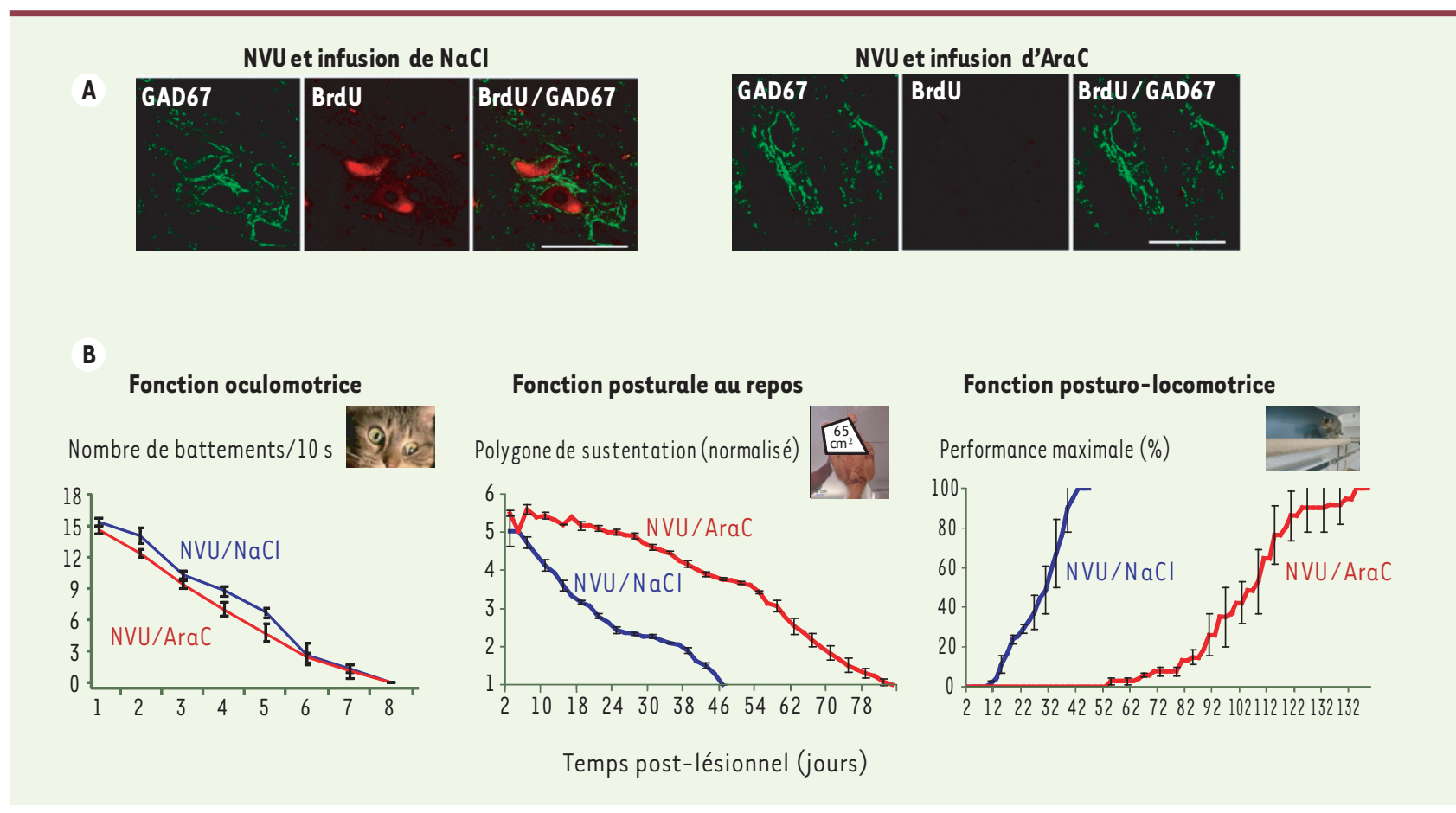

Figure 4. Illustration du rôle fonctionnel de la neurogenèse. A. Gauche : microphotographies illustrant de nouveaux neurones immunopositifs pour la GAD67 (vert) et pour le BrdU (rouge) dans le noyau vestibulaire médian désafférenté d'un chat après neurectomie vestibulaire unilatérale (NVU) et auquel $\mathrm{du} \mathrm{NaCl}$ a été administré pendant 30 jours. Droite : après NVU et infusion d'un agent antimitotique pendant 30 jours (cytosine- $\beta$-D arabinofuranoside: AraC), les neurones immunopositifs pour la GAD67 ne sont pas marqués au BrdU, indiquant l'absence de nouveaux neurones GABAergiques dans ce groupe d'animaux. Le BrdU est injecté 3 jours après NVU et les animaux sont perfusés 27 jours plus tard. Microscopie confocale, calibration $50 \mu \mathrm{m}$. B. Effets de l'infusion intracérébroventriculaire d'AraC sur la restauration des fonctions oculomotrice, posturale et locomotrice après NVU. Les courbes illustrent la valeur moyenne obtenue pour chaque groupe d'animaux. L'erreur standard de la moyenne est représentée par les barres verticales. Gauche : la fréquence du nystagmus est évaluée en fonction du nombre de battements rapides de l'œil de l'animal par unité de temps (10 secondes). Centre : les courbes illustrent la valeur moyenne de la surface du polygone de sustentation obtenue pour chaque groupe d'animaux. Les valeurs, mesurées en $\mathrm{cm}^{2}$, sont normalisées par rapport aux valeurs enregistrées avant la désafférentation, chaque animal constituant sa propre référence. Droite : la moyenne de la performance maximale d'équilibration cinétique (ordonnées) est exprimée en pourcentage de la performance pré-opératoire en fonction du temps post-lésionnel exprimé en jours (abscisses) pour les différents groupes d’animaux.

de l'importance du système GABAergique dans la compensation des déficits vestibulaires chez le mammifère adulte et signe l'existence de nouveaux mécanismes de plasticité adaptatifs post-lésionnels.

\section{Origine des nouvelles cellules et contrôle de leur intégration fonctionnelle dans les noyaux vestibulaires}

Les ventricules cérébraux hébergent des progéniteurs neuraux qui conserveraient au stade adulte leur capacité de proliférer et de se différencier en divers types neuronaux ou gliaux [4, 22]. Les NV, situés en bordure du IV ventricule, pourraient donc héberger des progéniteurs neuraux quiescents puisque chez le mammifère adulte, en conditions physiologiques, aucune prolifération cellulaire spontanée ne survient dans cette zone cérébrale. Ces cellules produites dans les NV pourraient également provenir de cellules épendymaires ou endothéliales présentes en bordure du IV ventricule ou encore de certains types d'astrocytes capables de se dédifférencier $[26,27]$. Une autre piste se dessine également: des progéniteurs pourraient migrer de la zone sous-ventriculaire jusqu'aux NV, en se déplaçant éventuellement le long de vaisseaux sanguins $[28,29]$.

Nous avons mis en évidence que la section unilatérale du nerf vestibulaire convertit les NV en une zone neurogène. L'émergence de cette neurogenèse réactionnelle pourrait s'expliquer par la rupture de l'homéostasie du microenvironnement cellulaire occasionnant le recrutement d'une cascade de mécanismes de neuroplasticité proneurogènes. En effet, nous avons observé dans notre modèle expérimental que la section et la dégénérescence rapide du nerf vestibulaire produisaient dans les NV désafférentés une augmentation des cellules micro- 


\section{PROCÉDURE CHIRURGICALE DE NEURECTOMIE VESTIBULAIRE UNILATÉRALE (NVU)}

La section du nerf vestibulaire (nerf VIII, vestibulocochléaire) est réalisée sous microscope opératoire selon une approche rétrosigmoïde dans des conditions d'asepsie rigoureuse. Les animaux sont anesthésiés à l'aide d'une solution de kétamine dihydrochloride injectée en intramusculaire (20 mg/kg, Rhône Poulenc, Mérieux, France) et reçoivent une injection d'analgésique (Tolfédine, 0,5 ml, Vetoquinol, Lure, France). Une couverture chauffante permet de maintenir l'animal anesthésié à une température comprise entre 37 et $38^{\circ} \mathrm{C}$. L'incision des tissus situés en arrière du pavillon auditif gauche permet l'accès à la bulla tympanique. La perforation de celle-ci à l'aide d'une fraise diamantée débouche sur l'oreille interne. La cavité labyrinthique est abordée par une ouverture creusée au-dessus des fenêtres ronde et ovale qui dévoile la VIII ${ }^{e}$ paire de nerfs crâniens. La section du nerf est pratiquée au niveau post-ganglionnaire où le nerf vestibulaire est séparé du nerf cochléaire, à mi-distance entre le ganglion de Scarpa et le tronc cérébral. Par la suite, le conduit auditif interne est obturé à l'aide de spongel cicatrisant et les tissus superficiels sont recousus. Les animaux sont placés sous antibiotiques pendant 6 jours et reçoivent des analgésiques pendant les 48 heures suivant la lésion. L'apparition du syndrome vestibulaire (symptômes oculomoteurs, posturaux et locomoteurs) au réveil de l'animal confirme cliniquement la réussite de la neurectomie vestibulaire unilatérale.

gliales et des astrocytes $[24,25]$, une augmentation de l'efficacité des systèmes GABAergique, cholinergique et histaminergique [20, 21, 26], ainsi qu'une augmentation du nombre de neurones exprimant le BDNF, le NGF, la neurotrophine 3 (NT3) et leurs récepteurs à activité tyrosine kinase respectifs (TrkA, TrkB et TrkC) [18]. Dans une étude récente, nous avons également observé que les glucocorticoïdes, connus pour entraver la neurogenèse [27], étaient régulés négativement dans les NV après NVU [28]. Ces résultats sont à rapprocher de données de la littérature mentionnant le rôle de ces facteurs respectifs dans la modulation des différentes étapes de la neurogenèse, allant de la prolifération des cellules souches neurales jusqu'à leur différenciation et intégration dans des circuits neuronaux complexes [8, 29-31]. D'autres facteurs actuellement en cours d'étude (facteurs de l'inflammation) également exprimés fortement dans les NV désafférentés [32] agiraient de concert pour promouvoir localement et ponctuellement un environnement neurogène dans les NV désafférentés.

\section{Neurogenèse des NV et restauration des fonctions posturo-locomotrices}

La présence inattendue de cette neurogenèse secondaire dans les NV désafférentés nous a également conduits à nous interroger sur son rôle fonctionnel dans la restauration des fonctions vestibulaires. Pour répondre à cette question, nous avons utilisé un agent antimitotique : la cytosine- $\beta-D$ arabinofuranoside (AraC) qui bloque la prolifération cellulaire dans les NV. Une solution d'AraC ou de chlorure de sodium a été administrée de manière continue dans le liquide céphalo-rachidien du IVe ventricule, en bordure des NV, durant 30 jours via une pompe osmotique Alzet à des chats ayant subi une NVU et chez des contrôles. Les conséquences cellulaires et comportementales du blocage de la neurogliogenèse par l'AraC ont été évaluées. Chez les animaux ayant reçu de l'AraC immédiatement après NVU (du $1^{\text {er }}$ au $30^{\circ}$ jour), l'activité mitotique a été totalement absente (diminution de cellules BrdU+de $99,66 \%$ dans le NVM, 99,91\% dans le NVI, $99,77 \%$ dans le NVL et $99,42 \%$ dans le NVS, correspondant respectivement aux noyaux vestibulaires médian [NVM], inférieur [NVI], latéral [NVL] et supérieur [NVS]) et la restauration des fonctions posturale et locomotrice a été considérablement retardée (trois mois au lieu d'un mois chez les animaux témoins) (voir Figure $4 A$ et $B$ ). Ces données témoignent de l'implication de la neurogenèse GABAergique et de l'astrogenèse dans les processus de restauration des fonctions posturo-locomotrices. Cependant des travaux complémentaires sont nécessaires pour affirmer le lien de causalité entre neurogenèse et compensation vestibulaire.

En revanche, la rapide récupération de la fonction oculomotrice (6 jours) n'a pas été altérée par l'infusion d'AraC. Ceci suggère que la compensation du nystagmus oculaire horizontal spontané reposerait sur d'autres mécanismes de plasticité, qui interviendraient temporellement en amont de la neurogenèse et la gliogenèse [25]. En adéquation avec cette hypothèse, les travaux d'une équipe de chercheurs néo-zélandais ont montré qu'après lésion vestibulaire unilatérale, l'infusion d'oligonucléotides antisens bloquant l'expression du BDNF (facteur favorisant entre autres la neurogenèse) chez la souris conduisait à un retard dans la compensation des déficits posturaux, sans incidence sur le nystagmus oculaire [33]. Dans une autre étude, l'infusion chronique d'antagonistes des récepteurs $G A B A_{A}$ dans les NV désafférentés a modifié l'expression des symptômes posturaux chez le cochon d'Inde, sans altérer l'expression des symptômes oculomoteurs [34]. Ainsi la compensation des paramètres posturolocomoteurs et oculomoteurs serait gouvernée par des mécanismes de plasticité différents.

\section{Le GABA : un acteur principal du retour à l'homéostasie des noyaux vestibulaires}

La littérature sur la compensation vestibulaire mentionne depuis de nombreuses années l'importance du système GABAergique dans la restauration des fonctions vestibulaires [35]. Dans notre modèle de NVU, 
la présence d'une neurogenèse GABAergique fonctionnelle est un résultat original qui attribue au système GABAergique un rôle pivot dans la récupération des déficits survenant après atteinte vestibulaire. Le système GABAergique est en effet connu pour contribuer de manière importante au rééquilibrage de l'activité électrique entre les NV homologues [15]. Nous proposons que les nouveaux neurones GABAergiques appartenant aux NV désafférentés pourraient inhiber localement des neurones inhibiteurs, rehaussant ainsi l'excitabilité intrinsèque. Une autre hypothèse serait que des réorganisations du milieu extracellulaire puissent conduire à une réexpression ponctuelle de cotransporteurs de cations et de chlorures $\mathrm{Na}^{+} \mathrm{K}^{+} 2 \mathrm{Cl}^{-}$de type 1 ( $\mathrm{NKCCl}$ ). En effet, dans les premiers stades du développement et suite à certaines lésions engendrant une neurogenèse réactionnelle chez l'adulte, le GABA peut avoir une action dépolarisante sur les cellules pourvues de récepteurs de type $\mathrm{GABA}_{A}$ et de NKCCI $[36,37]$. Dans ces conditions particulières, la présence de NKCCl à la surface des membranes neuronales conduit à une accumulation intracellulaire de $\mathrm{Cl}^{-}$. Par conséquent, l'ouverture d'un récepteur ionotropique $\mathrm{GABA}_{A}$ induit une sortie d'ions $\mathrm{Cl}^{-}$créant ainsi un flux dépolarisant. Un tel phénomène pourrait ainsi se produire en réaction à la NVU et favoriser l'augmentation progressive de l'activité électrique des neurones des NV désafférentés au cours de la compensation vestibulaire.

\section{Conclusions}

Nos données originales et novatrices dans le domaine de la compensation vestibulaire pourraient offrir de nouvelles pistes en clinique humaine. Les différentes pathologies du système vestibulaire sont extrêmement invalidantes (vertiges, nausées, vomissements, pertes d'équilibre et de repères spatiaux). Comprendre comment le système nerveux central s'adapte et met en place une récupération comportementale après une lésion est un préalable au choix de traitements ciblés et efficaces, ainsi qu'à la mise en œuvre de meilleures procédures de réhabilitation.

Notre modèle expérimental, désafférentation vestibulaire induisant une neurogenèse fonctionnelle dans les noyaux vestibulaires du mammifère adulte, permet de déterminer les facteurs permettant à une zone cérébrale non neurogène de pouvoir ponctuellement produire de nouveaux neurones. Cependant, nous avons récemment démontré que la neurogenèse réactionnelle ne survenait qu'après désafférentation structurale et fonctionnelle des NV (NVU), alors qu'elle demeure inexistante après une désafférentation fonctionnelle plus progressive (labyrinthectomie: destruction chirurgicale des récepteurs labyrinthiques) [38]. La neurectomie vestibulaire reproduit des pathologies vestibulaires de survenue rapide (névrite vestibulaire, neurinome de l'acoustique, chirurgie du Ménière) tandis que la labyrinthectomie est plus proche d'un processus progressif de vieillissement des récepteurs vestibulaires périphériques, de leur lésion par traumatisme crânien ou de leur intoxication par des antibiotiques. Ainsi, il apparaît que les mécanismes de compensation vestibulaire dépendent étroitement de l'étiologie du trouble. Une meilleure connaissance de ces mécanismes permettrait de mieux cibler la pharmacologie et la rééducation fonctionnelle en pathologie vestibulaire. $\diamond$

\section{SUMMARY}

Discovering a new functional neurogenic zone : the vestibular nuclei of the brainstem

The adult mammal brain is mostly considered as nonneurogenic, except in the subventricular zone of the lateral ventricles and the subgranular zone of the dentate gyrus, where ongoing neurogenesis occurs. However, anti-neurogenic influences can be removed in pathological conditions or after specific injury. That is what happens in a model of unilateral vestibular neurectomy (UVN) that mimics human pathology in adult cats. We showed for the first time that a UVN promoted an intense reactive cell proliferation in the deafferented vestibular nuclei located in the brainstem. The new cells survived up to one month, differentiated into glial cells - microglia or astrocytes - or GABAergic neurons, so highlighting a GABAergic neurogenesis. Surprisingly, we further showed that post-UVN reactive cell proliferation contributed successfully to fine restoration of vestibular posturo-locomotor functions. In conclusion, these pioneering studies bring new pieces of a promising puzzle in both stem cell and vestibular therapy domains. $\diamond$

\section{CONFLITS D'INTÉRÊTS}

Les auteurs déclarent n'avoir aucun conflit d'intérêts concernant les données publiées dans cet article.

\section{RÉFÉRENCES}

1. Zhao C, Deng W, Gage FH. Mechanisms and functional implications of adult neurogenesis. Cell $2008 ; 132:$ 645-60.

2. Gage FH, Coates PW, Palmer TD, et al. Survival and differentiation of adult neuronal progenitor cells transplanted to the adult brain. Proc Natl Acad Sci USA $1995 ; 92$ : 11879-83.

3. Lie DC, Dziewczapolski G, Willhoite AR, et al. The adult substantia nigra contains progenitor cells with neurogenic potential. J Neurosci $2002 ; 22$ : 6639-49.

4. Shihabuddin LS, Horner PJ, Ray J, et al. Adult spinal cord stem cells generate neurons after transplantation in the adult dentate gyrus. J Neurosci 2000 ; $20: 8727-35$.

5. Herrera DG, Garcia-Verdugo JM, Alvarez-Buylla A. Adult-derived neural precursors transplanted into multiple regions in the adult brain. Ann Neurol $1999 ; 46: 867-77$.

6. Suhonen J0, Peterson DA, Ray J, et al. Differentiation of adult hippocampusderived progenitors into olfactory neurons in vivo. Nature $1996 ; 383: 624-7$.

7. Emsley JG, Mitchell BD, Kempermann G, et al. Adult neurogenesis and repair of the adult CNS with neural progenitors, precursors, and stem cells. Prog Neurobiol $2005 ; 75: 321-41$.

8. Moyse $\varepsilon$, Segura S, Liard 0 , et al. Microenvironmental determinants of adult neural stem cell proliferation and lineage commitment in the healthy and injured central nervous system. Curr Stem Cell Res Ther 2008 ; 3 : 163-84.

9. Taupin $P$, Neurogenesis in the pathologies of the nervous system. Med Sci (Paris) $2005 ; 21: 711-4$.

10. Lacour $M$, Restoration of vestibular function : basic aspects and practical advances for rehabilitation. Curr Med Res Opin 2006 ; 22 : 1651-9. 


\section{RÉFÉRENCES}

11. Ris L, de Waele C, Serafin M, et al. Neuronal activity in the ipsilateral vestibular nucleus following unilateral labyrinthectomy in the alert guinea pig. J Neurophysiol $1995 ; 74: 2087-99$.

12. Zennou-Azogui Y, Borel L, Lacour M, et al. Recovery of head postural control following unilateral vestibular neurectomy in the cat. Neck muscle activity and neuronal correlates in Deiters' nuclei. Acta Otolaryngol Suppl $1993 ; 509$ : 1-19.

13. Borel L, Lopez C, Peruch $\mathrm{P}$, et al. Vestibular syndrome : a change in internal spatial representation. Neurophysiol Clin. $2008 ; 38: 375-89$

14. Campos Torres A, Vidal PP, de Waele C. Evidence for a microglial reaction within the vestibular and cochlear nuclei following inner ear lesion in the rat. Neuroscience $1999 ; 92: 1475-90$.

15. Darlington CL, Smith PF. Molecular mechanisms of recovery from vestibular damage in mammals : recent advances. Prog Neurobiol $2000 ; 62: 313-25$

16. de Waele $\mathrm{C}$, Campos Torres $\mathrm{A}$, Josset $\mathrm{P}$, et al. Evidence for reactive astrocytes in rat vestibular and cochlear nuclei following unilateral inner ear lesion. Eur J Neurosci $1996 ; 8: 2006-18$.

17. Gacek RR, Khetarpal U, Schoonmaker J. Morphological and neurochemical correlates of vestibular compensation. Auris Nasus Larynx 1998 ; 25 : 193-201.

18. Lacour M, Tighilet $B$. Plastic events in the vestibular nuclei during vestibular compensation : the brain orchestration of a "deafferentation" code. Restor Neurol Neurosci 2010 ; 28 : 19-35.

19. Paterson JM, Short D, Flatman PW, et al. Changes in protein expression in the rat medial vestibular nuclei during vestibular compensation. J Physiol $2006 ; 575: 777-88$

20. Tighilet $B$, Lacour M. Gamma amino butyric acid (GABA) immunoreactivity in the vestibular nuclei of normal and unilateral vestibular neurectomized cats. Eur J Neurosci $2001 ; 13: 2255-67$.

21. Tighilet $B$, Lacour $M$. Distribution of choline acetyltransferase immunoreactivity in the vestibular nuclei of normal and unilateral vestibular neurectomized cats. Eur J Neurosci $1998 ; 10: 3115-26$.

22. Martens DJ, Seaberg RM, van der Kooy D. In vivo infusions of exogenous growth factors into the fourth ventricle of the adult mouse brain increase the proliferation of neural progenitors around the fourth ventricle and the central canal of the spinal cord. Eur J Neurosci $2002 ; 16: 1045-57$.

23. Bauer $S$, Hay M, Amilhon B, et al. In vivo neurogenesis in the dorsal vagal complex of the adult rat brainstem. Neuroscience $2005 ; 130: 75-90$.

24. Tighilet B, Brezun JM, Sylvie GD, et al. New neurons in the vestibular nuclei complex after unilateral vestibular neurectomy in the adult cat. Eur J Neurosci 2007 ; $25: 47-58$.

25. Dutheil S, Brezun JM, Leonard J, et al. Neurogenesis and astrogenesis contribution to recovery of vestibular functions in the adult cat following unilateral vestibular neurectomy : cellular and behavioral evidence. Neuroscience 2009 ; 164 : 1444-56.

26. Tighilet $B$, Trottier $S$, Mourre $C$, et al. Changes in the histaminergic system during vestibular compensation in the cat. J Physiol 2006; $573: 723-39$

27. Gould $\varepsilon$, Tanapat P, Stress and hippocampal neurogenesis. Biol Psychiatry $1999 ; 46: 1472-9$.
28. Tighilet B, Manrique C, Lacour M. Stress axis plasticity during vestibular compensation in the adult cat. Neuroscience. $2009 ; 160: 716-30$.

29. Pathania M, Yan LD, Bordey A. A symphony of signals conducts early and late stages of adult neurogenesis. Neuropharmacology $2010 ; 58: 865-76$.

30. Mathieu P, Battista D, Depino A, et al. The more you have, the less you get : the functional role of inflammation on neuronal differentiation of endogenous and transplanted neural stem cells in the adult brain. J Neurochem $2010 ; 112: 1368-85$.

31. Molina-Hernandez A, Velasco I. Histamine induces neural stem cell proliferation and neuronal differentiation by activation of distinct histamine receptors. J Neurochem 2008 ; 106 : 706-17.

32. Liberge M, Manrique C, Bernard-Demanze L, et al. Changes of TNF-alpha, NFKB and MnSOD protein in the vestibular nuclei following a unilateral vestibular deafferentation. J Neuroinflammation $2010 ; 7: 91$.

33. Bolger C, Sansom AJ, Smith PF, et al. An antisense oligonucleotide to brain-derived neurotrophic factor delays postural compensation following unilateral labyrinthectomy in guinea pig. Neuroreport. $1999 ; 10: 1485-8$.

34. Gliddon CM, Darlington CL, Smith PF. Effects of chronic infusion of a GABAA receptor agonist or antagonist into the vestibular nuclear complex on vestibular compensation in the guinea pig. J Pharmacol Exp Ther $2005 ; 313$ : 1126-35.

35. Gliddon CM, Darlington CL, Smith PF. GABAergic systems in the vestibular nucleus and their contribution to vestibular compensation. Prog Neurobio $2005 ; 75: 53-81$

36. Ge S, Goh EL, Sailor KA, et al. GABA regulates synaptic integration of newly generated neurons in the adult brain. Nature $2006 ; 439$ : 589-93.

37. Rivera C, Voipio J, Payne JA, et al. The $\mathrm{K}+/ \mathrm{Cl}$ - co-transporter $\mathrm{KCC} 2$ renders GABA hyperpolarizing during neuronal maturation. Nature 1999 ; $397: 251-5$.

38. Lacour M, Dutheil S, Tighilet B, Lopez C, Borel L. Tell me your vestibular deficit, and i'll tell you how you'll compensate. Ann N Y Acad Sci 2009; $1164: 268-78$

39. de Chevigny A, Lledo PM. Olfactory bulb neurogenesis and its neurological impact. Med Sci (Paris) $2006 ; 22: 607-13$

\section{TIRÉS À PART}

B. Tighilet

\section{Bon de commande}

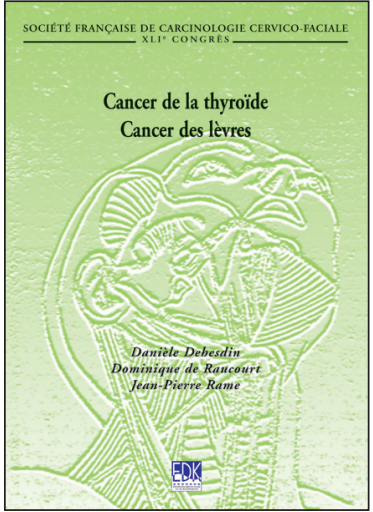

ISBN : 978-2-8425-4137-8 264 pages
À retourner à EDK, 2, rue Troyon - 92316 Sèvres Cedex

Tél. : 0155641393 - Fax : 0155641394 - E-mail : edk@edk.fr

NOM :

Prénom :

Adresse :

Code postal :

Ville :

Pays :

Fonction :

Je souhaite recevoir l'ouvrage Cancer de la thyroïde - Cancers des lèvres : $35 €+3 €$ de port $=38 € \mathbf{T T C}$

en ................. exemplaire, soit un total de ....................................... €

$\square$ Par chèque, à l'ordre de $\mathbf{E} \mathbf{D} \mathbf{K}$

$\square$ Par carte bancaire:

$\square$ Visa $\square$ Eurocard/Mastercard

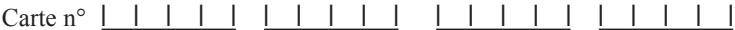

Date d'expiration: $\quad$ | 1 | 1 |

$\mathrm{N}^{\circ}$ de contrôle au dos de la carte : $\quad \underline{|\quad| \quad \mid}$ 